\title{
Emergency dental care triage during the COVID-19 pandemic
}

\author{
Madeleine Ball, ${ }^{1}$ Dapo Akintola, ${ }^{2}$ Zoe Harrington ${ }^{3}$ and Serpil Djemal ${ }^{\star 4}$
}

\section{Key points}

Rapid and up-to-date service transformation, as well as sufficient local provision of services via Urgent Dental Care hubs, is essential to respond to public health challenges.
Certain dental conditions can be easily managed with advice over the phone, such as temporomandibular joint dysfunction, while telephone triage is an effective way of prioritising emergency patients for a face-to-face appointment.
A secondary care dental hospital provides a good setting to cope with a significant proportion of the dental needs during a pandemic owing to its large number of dental chairs, staff from a range of disciplines and an appropriate administrative team.

\begin{abstract}
Introduction Due to the COVID-19 pandemic, from 23 March 2020, routine dental treatment was stopped by the Chief Dental Officer, with the emphasis towards urgent dental care only.
\end{abstract}

Aim To evaluate the activities of the emergency service at a secondary care Urgent Dental Care (UDC) hub during the COVID-19 pandemic.

Materials and methods The total number of patients seen from 30 March to 20 June 2020 was recorded. The effectiveness of telephone triage and the appropriateness of patients invited for a clinical assessment were evaluated over a two-week period.

Results The number of calls into the UDC hub were highest during the first few weeks, with up to 249 per day. The most commonly provided emergency treatments included extractions and pulp extirpations.

Discussion As other UDC hubs opened, the number of calls reduced, with patients being directed to a UDC nearer to where they lived.

Conclusions The dental profession had to make some significant changes in the way they worked due to the risk of COVID-19 transmission and due to the effects of the lockdown. This review highlights the effectiveness of telephone triage as well as its drawbacks.

\section{Introduction}

Due to the lockdown as a result of the COVID-19 pandemic from 23 March 2020, the emphasis in oral healthcare shifted away from the delivery of routine dental care to urgent care only. Urgent Dental Care (UDC) hubs were established at short notice to receive patients with dental emergencies.

The emergency service at King's College Hospital was previously a walk-in service, where

'Dental Core Trainee, King's College Hospital, UK; ${ }^{2}$ Consultant in Oral Surgery, King's College Hospital

UK; ${ }^{3}$ Consultant in Restorative Dentistry, King's College Hospital, UK; ${ }^{4}$ Consultant in Restorative Dentistry, King's College Hospital, King's College NHS Foundation Trust,

Bessemer Road, Camberwell, London, SE5 9RW, UK.

*Correspondence to: Serpil Djemal

Email address: serpil.djemal@nhs.net

Refereed Paper.

Accepted 19 November 2020

https://doi.org/10.1038/s41415-021-3379-z patients would queue for an appointment, but in August 2019, it evolved into an appointment service in response to patient feedback, with 40 appointments being available and the first 40 patients calling in receiving an appointment. This service saw approximately 200 patients per week from across London.

This call-in service was converted into a UDC hub during the pandemic, with a telephone triage system developed to identify patients requiring emergency dental care and invite them in for a face-to-face consultation. The pathway was set up to minimise unnecessary footfall through the hospital and help maintain social distancing rules, thereby reducing the potential for virus transmission.

The hub was led by an oral surgeon and had staff from restorative, paediatrics, orthodontics and special care dentistry departments. There was no shortage of staff since all routine clinics were cancelled. During the periods when the data were collected, telephone triage was provided by NHS consultants using seven telephones. Triage ran daily from 8 am until 4 pm, Monday to Friday.

As outlined in the Chief Dental Officer's (CDO's) letter of preparedness, ${ }^{1}$ emergency conditions which met the acceptance criteria for a clinical assessment included serious and lifethreatening conditions as well as uncontrollable pain, swelling and trauma. Patients involved in trauma and those with swollen faces were invited to send photographs to an NHS e-mail address, and in situations where there was any uncertainty over the telephone, photographs helped confirm whether to invite the patient for a clinical assessment.

The clinicians involved also gave advice about analgesics and how to cope with nonurgent problems, as well as prescribing antibiotics when necessary. Patients who were to be seen for clinical assessment were 
also triaged for vulnerability and potential infection with COVID-19 so they could be seen in clearly identified and separate areas of the hospital.

\section{Aims and objectives}

The aim of this service review is to evaluate the activities of the emergency service at a secondary care UDC hub during the COVID-19 pandemic. Firstly, it assesses the total number of patients seen from 30 March to 20 June 2020. Secondly, it looks at the effectiveness of telephone triage over two separate weeks, one month apart. In addition, the appropriateness of the patients invited for a clinical assessment was evaluated by looking at the patients' signs and symptoms recorded on the triage notes and whether these matched the signs and symptoms recorded at the clinical assessment. The patient demographics and the type of treatments provided were also analysed.

\section{Methodology}

The total number of patients contacting the service and the number of patients invited to attend for a clinical assessment were recorded from 30 March to 20 June 2020.
The triage forms and the clinical records were retrospectively scrutinised and analysed for patients seen for clinical assessment during the weeks commencing 6 April 2020 and 11 May 2020. The anticipation was that we would be able to compare activity early on in the lockdown, when the service had just transitioned into an UDC hub, with activity one month later to see any changes in trends such as patient demographics and treatment provided.

\section{Data collection}

During the whole timeframe from 30 March to 20 June 2020, the following data were collected:

- Total numbers of patients receiving telephone triage

- Total number of patients receiving clinical assessment.

During the two weeks chosen for data analysis in April and May, the following data were collected:

- Total numbers of failures to attend

- Total numbers of repeat triages

- Total numbers of repeat attendances

- Patient demongraphics:
o Age
o Gender
o Home postcode

Table 1 Patient contact activity via telephone triage and attendance for clinical assessment

\begin{tabular}{l|l|l|l} 
Date & Telephone triage & Clinical assessment & $\begin{array}{l}\text { Percentage of callers offered an } \\
\text { appointment (\%) }\end{array}$ \\
\hline $30 / 03 / 2020$ & 518 & 165 & 31.9 \\
\hline $06 / 04 / 2020$ & 835 & 162 & 19.4 \\
\hline $13 / 04 / 2020$ & 943 & 244 & 25.9 \\
\hline $20 / 04 / 2020$ & 678 & 166 & 17.6 \\
\hline $27 / 04 / 2020$ & 582 & 177 & 30.4 \\
\hline $04 / 05 / 2020$ & 551 & 173 & 31.4 \\
\hline $11 / 05 / 2020$ & 693 & 219 & 31.6 \\
\hline $18 / 05 / 2020$ & 619 & 63 & 10.2 \\
\hline $26 / 05 / 2020$ & 528 & 126 & 23.9 \\
\hline $01 / 06 / 2020$ & 592 & 205 & 34.6 \\
\hline $08 / 06 / 2020$ & 545 & 145 & 26.6 \\
\hline $15 / 06 / 2020$ & 364 & 133 & 36.5 \\
\hline
\end{tabular}

- Triage data:

o Triage date and appointment date

o Whether use of analgesics and antibiotics had been recorded on the triage form

o Whether the patient was medically compromised or vulnerable

- Clinical data:

o Whether the triage information matched the clinical information

o Whether the patients offered a clinical assessment fulfilled the criteria for an appointment

o Diagnosis

o Treatment provided.

\section{Results}

During the timeframe from 30 March to 20 June 2020, a total of 7,448 patients called the telephone triage service at King's College Hospital. Of these, 1,978 patients were invited to attend for a clinical assessment and the weekly breakdown of activity, as well as the percentage of callers invited to attend for a clinical assessment, can be seen in Table 1.

Data from the week commencing 6 April (period 1) reveal 162 of the total 835 callers were invited for a clinical assessment, of which three patients failed to attend; therefore, 159 patients' notes were analysed. By comparison, data from the week commencing 11 May (period 2) revealed 219 of the total 693 callers were invited for clinical assessment, of which four patients failed to attend; therefore, 215 patients' notes were analysed (Table 2).

During period $1,91.8 \%$ of patients were seen on the same day that they called, compared to $83.7 \%$ of patients in period 2 (Table 2 ).

Of the 159 patients who attended for a clinical assessment in period 1, eight (5\%) had previously contacted the triage service and received advice during the COVID-19 period.

Four patients (2.5\%) had previously attended for a clinical assessment. By comparison, during period 2, 21 patients $(9.8 \%)$ contacted the triage service for a second time and 21 patients $(9.8 \%)$ were seen for a second clinical appointment, as illustrated in Table 2.

Table 2 Total number of patients seen and breakdown of attendance

\begin{tabular}{|l|l|l|l|l} 
Week commencing & $\begin{array}{l}\text { Number of } \\
\text { patients seen }\end{array}$ & $\begin{array}{l}\text { Number of patients seen on the } \\
\text { same day as their telephone triage }\end{array}$ & $\begin{array}{l}\text { Number of patients calling } \\
\text { for a second time }\end{array}$ & $\begin{array}{l}\text { Number of patients attending for } \\
\text { a second clinical assessment }\end{array}$ \\
\hline 6 April (period 1) & 159 & $146(91.8 \%)$ & $8(5 \%)$ & $4(2.5 \%)$ \\
\hline 11 May (period 2) & 215 & $180(83.7 \%)$ & $21(9.8 \%)$ & $21(9.8 \%)$ \\
\hline
\end{tabular}


Twelve patients (7.5\%) seen during period 1 were medically compromised or vulnerable compared to 19 (8.8\%) during period 2.

\section{Patient demographics}

The age range of patients that received clinical assessment in period 1 was $17-83$ years and in period 2 was $16-84$ years. Patients above 70 years of age were directed to a separate waiting area and were seen on a 'vulnerable' clinic with enclosed bays.

The gender distribution for both periods can be seen in Figure 1, showing 73 men and 86 women accessed the service during period 1 and 107 men and 108 women during period 2 .

An attempt was made to ascertain how far patients had travelled to access emergency care by plotting their postcodes using Google Maps (Fig. 2). While the majority of the patients were centred around Greater London, a small number travelled from as far as Hastings, Brighton and Portsmouth.

\section{Use of analgesics and antibiotics} recorded on the telephone triage form

In general, only patients who had already taken appropriate analgesics and antibiotics (if indicated) with no relief of their symptoms met the criteria to attend for a face-to-face clinical consultation. Whether this was the case was looked at restrospectively on the telephone triage form.

During period 1, 125 patients $(78.6 \%)$ reported taking the correct analgesics but with inadequate relief of their pain and 34 (11.4\%) recorded not having taken any. Sixty-six patients (41.5\%) reported having had at least one course of antibiotics with no impact on their symptoms, with 93 (58.5\%) not having taken any. By comparison, during period 2, 135 patients (62.7\%) reported taking the correct analgesic but with inadequate relief of their pain and $80(37.3 \%)$ recorded not having taken any. One hundred and two patients (47.4\%) reported having had at least one course of antibiotics with no impact on their symptoms, with 113 (52.6\%) not having taken any.

\section{Diagnoses}

During periods 1 and 2, apical periodontitis was responsible for the majority of the emergency cases, with 47 patients $(29.6 \%)$ with acute cases and 44 patients $(27.7 \%)$ with chronic cases in period 1 and $45(20.9 \%)$ and 87 (40.5\%), respectively, in period 2 (Table 3 ).

\section{Fig. 1 The ratios of men to women seen for clinical assessment during both periods}
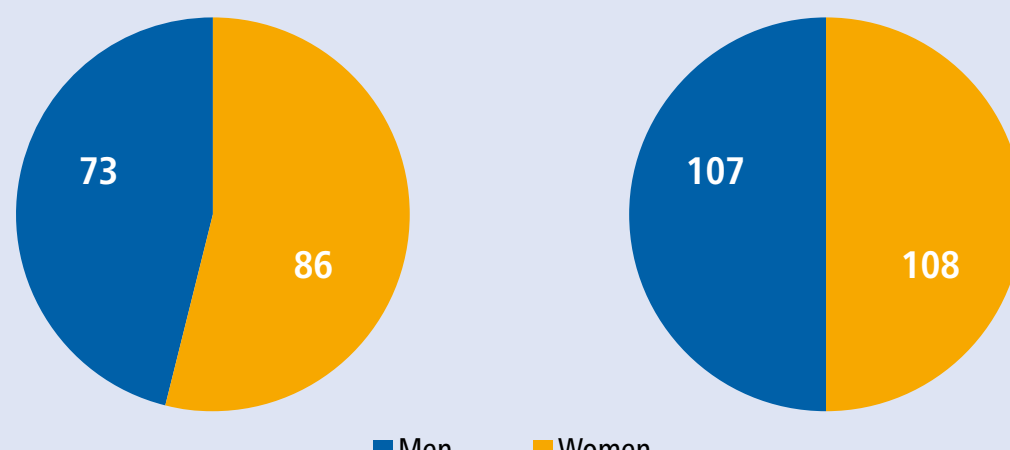

- Men

Women
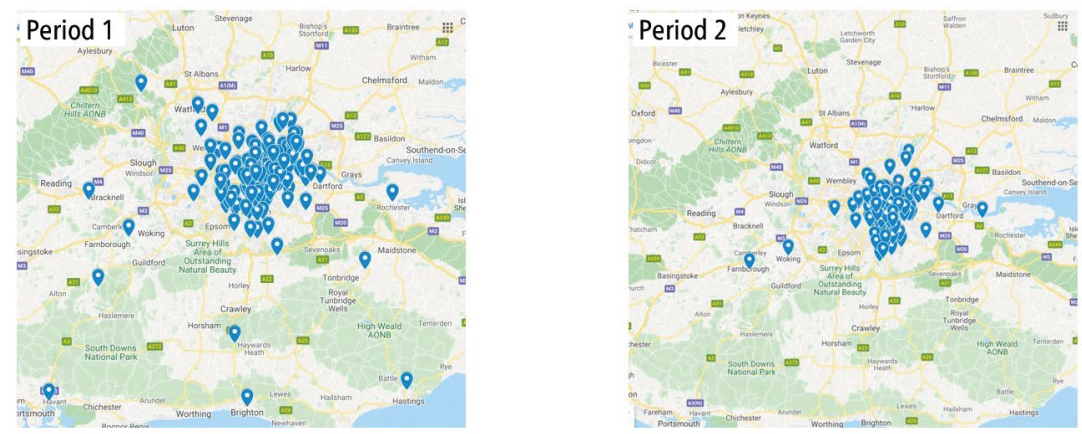

Fig. 2 Patient postcodes entered into Google Maps to ascertain the distance travelled for a clinical assessment during periods 1 and 2 (data credit line: Map data ( 2020 Google)

There was little difference in the number of patients diagnosed with irreversible pulpitis, periodontal abscess and fractured teeth between periods 1 and 2 (Table 3 ). There were, however, more cases of dental trauma during period 2.

\section{Treatment provided}

The most common treatment modality was extraction, with 119 patients receiving one or more extractions during period 1 and 154 patients during period 2. Pulp extirpations were the next most common, with ten patients in period 1 and 21 patients in period 2 having at least one tooth extirpated (Fig. 3).

During period 1, three patients $(1.9 \%)$ were reassured and given advice only, while 11 patients $(6.9 \%)$ declined the treatment offered (Fig. 3). By comparison, during period 2 , five patients $(2.3 \%)$ were reassured and given advice only, while nine patients (4.2\%) declined the treatment offered (Fig. 3).

During period 2, there were some additional treatments carried out that did not occur in period 1 (Table 4).

During period 1, all of the 159 patients who were offered a clinical assessment fulfilled the acceptance criteria for emergency care. When comparing their signs and symptoms reported over telephone triage with those reported at the clinical assessment, this was consistent in 157 (98.7\%) patients.

During period 2, one patient $(0.5 \%)$ had a loose bridge, which did not fulfil the acceptance criteria for emergency care. When comparing their signs and symptoms reported over telephone triage with those reported at clinical assessment, this was consistent in 208 (96.7\%) patients.

\section{Discussion}

The figures for telephone triage were highest during the first three weeks of operation of the secondary care UDC hub, with call volumes of up to 249 per day and 943 per week. King's College Hospital received such large numbers of calls because all dental practices were instructed to close by the CDO, but there were initially too few UDC hubs open to enable patients to be seen local to their home address. As well as this, the telephone number for the King's emergency dental service was online and so we received referrals from closed 


\begin{tabular}{|c|c|c|}
\hline Diagnoses & $\begin{array}{l}\text { Period } 1 \\
6-11 \text { April }\end{array}$ & $\begin{array}{l}\text { Period } 2 \\
11-15 \text { May }\end{array}$ \\
\hline Acute apical periodontitis & 47 & 45 \\
\hline Chronic apical periodontitis & 44 & 87 \\
\hline Irreversible pulpitis & 22 & 25 \\
\hline Periodontal abscess & 20 & 18 \\
\hline Fractured tooth & 8 & 10 \\
\hline Vertical root fracture & 3 & 1 \\
\hline Temporomandibular joint dysfunction & 3 & 5 \\
\hline Pericoronitis & 3 & 0 \\
\hline Dental trauma & 1 & 10 \\
\hline Failed extraction & 1 & 0 \\
\hline Debonded splint & 1 & 0 \\
\hline Dentine hypersensitivity & 1 & 0 \\
\hline Root resorption & 1 & 0 \\
\hline Alveolar osteitis & 1 & 0 \\
\hline Radicular cyst & 1 & 0 \\
\hline Bacterial sialadenitis & 1 & 1 \\
\hline Reversible pulpitis & 1 & 2 \\
\hline Lost crown, loose bridge, loose onlay & 0 & 3 \\
\hline Food packing & 0 & 2 \\
\hline Alveolar osteitis & 0 & 1 \\
\hline Sebaceous cyst & 0 & 1 \\
\hline Odontogenic keratocyst & 0 & 1 \\
\hline Infected cemento-osseous dysplasia & 0 & 1 \\
\hline Median rhomboid glossitis & 0 & 1 \\
\hline Tongue trauma due to a sharp tooth & 0 & 1 \\
\hline
\end{tabular}

practices across London and further afield. The 111 service also provided the telephone number and some patients reported being given the number by their dentist, or from their dental practice's website.

The number of calls reduced after three weeks, owing to the opening of other UDC hubs and the introduction of the 'out of area' guidance which enabled each UDC hub to cover a specific area. Due to the introduction of this guidance, fewer patients travelled from afar to reach the service during the week in May (period 2) compared to the week in April (period 1) because the UDC hub would only accept patients from certain areas, directing others to their local service.

As a member of staff who worked on the emergency service before and during COVID-19, there was a shift in the type of patient presentation. Beforehand, a significant proportion of patients were not registered with a dental practitioner and presented with extensive caries and periodontal disease. Many had South London postcodes, and according to the Indices of Deprivation 2019, many of the surrounding areas of King's such as Lambeth and Croydon are ranked withinin the top $10 \%$ of the most deprived areas nationally. ${ }^{2}$ However, during the pandemic, patients attended from all over London and most patients had their own dental practitioner who was closed. More isolated problems were seen such as pain from a part-completed root canal treatment and pulpal necrosis from a deep restoration in a relatively well-cared-for dentition.

For periods 1 and 2, over $99 \%$ of patients met the criteria for an urgent appointment according to the telephone triage signs and

Fig. 3 Graphs to show the type of treatments provided across the two periods
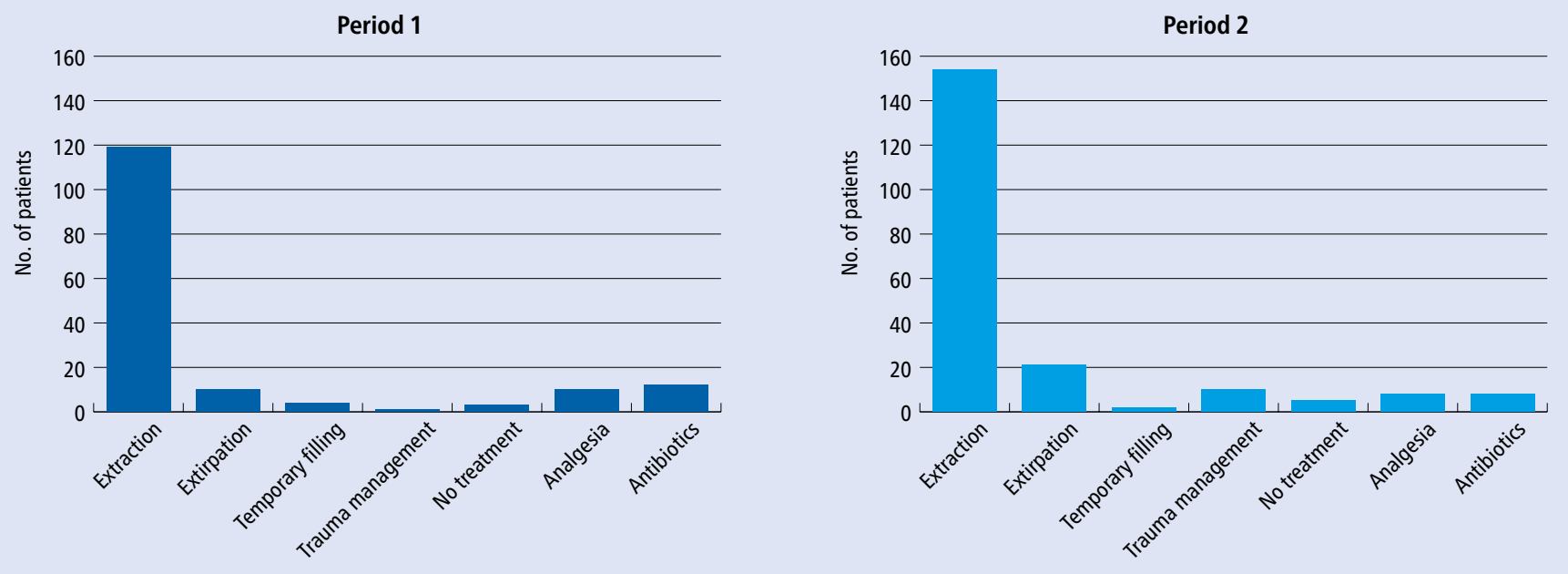
Table 4 Some of the less common treatments provided during period 2

\begin{tabular}{l|l}
\hline Treatment & Quantity \\
\hline Re-cement crown/bridge/onlay & 3 \\
\hline Oral hygiene advice & 2 \\
\hline Drain cyst & 1 \\
\hline Irrigation and Alvogyl dressing & 1 \\
\hline
\end{tabular}

symptoms. This was below $100 \%$ for period 2 because one patient was given an appointment for a loose bridge. For both periods, over $96 \%$ of telephone triage signs and symptoms matched clinical signs and symptoms. These high figures show that a large proportion of patients who were offered an appointment required emergency treatment and therefore telephone triage was effective at identifying these patients.

However, the data show a mismatch between the telephone triage and clinical signs and symptoms for seven patients who were treated for non-urgent conditions. These include oral hygiene advice to prevent food packing, mild symptoms of reversible pulpitis and re-cementation of indirect restorations. One patient attended three times to have a bridge re-cemented, which evidently did not meet the acceptance criteria and shows how telephone triage relies on the honesty and accuracy of patients' descriptions.

A number of patients were managed with analgesia and advice for temporomandibular joint dysfunction and pericoronitis. We have learnt that with an accurate telephone triage, certain conditions can be managed over the phone to avoid the patient making the journey.

During periods 1 and 2, 11 (6.9\%) and nine (4.2\%) patients declined treatment, respectively. The reasons for this include patient wishes for a general anaesthetic or hoping to save an unrestorable tooth.

The data show that antibiotics were the primary management for 12 patients during period 1 and eight during period 2. This does not account for antibiotics prescribed in addition to extractions and antibiotics prescribed remotely. Initially, we directed patients to their local dental or medical practitioner because we were unable to access FP10 prescriptions. However, we learnt that remote prescribing is most efficiently and safely done by e-mailing the prescription to the patient's local pharmacy. We stopped prescribing remotely when the $\mathrm{CDO}$ asked practices to reopen.

The re-attendance rates were four patients (2.5\%) for period 1 and 21 (9.8\%) for period 2, and the range was $1-3$ times. The reasons include: patients with continued unmanageable pain after initially declining treatment due to anxiety; wishes to save an unrestorable tooth; alveolar osteitis; repeat extirpations; patients who first received antibiotics and later extraction for pericoronitis; those with multiple carious teeth; and dental traumas. There were three (1.9\%) and ten (4.7\%) dental traumas during periods 1 and 2, respectively. During the lockdown, more people cycled, and on 13 May 2020, the government allowed unlimited exercise which may have contributed to the increased rates of dental traumas during period 2 compared to period 1 .

The emergency dental service provided during COVID-19 evolved to keep up-to-date with new emerging guidance. The service stepped down as a UDC hub on 19 June 2020, enabling elective treatments to resume. With a second spike of COVID-19 infections and another lockdown, the service is now well prepared with bespoke pathways to ensure safe and effective patient care. While not ideal for all patients to travel to, a dental hospital offers a large number of dental chairs, access to staff from a range of disciplines and an appropriate administrative team to be able to cope with a significant proportion of dental needs during a pandemic. Moving forwards, ensuring there are sufficient UDC hubs to enable patients to be treated locally will help contain the virus and ensure patients have prompt access to emergency care.

\section{Key lessons learnt}

They key lessons learnt are:

- There should be improved communication and consistent information disseminated to UDC hubs, dental practitioners and 111. With improved leadership from government representatives, patient information about these services should be kept up-to-date and clear so patients can seek appropriate and timely help in the event of a dental emergency

- During the initial weeks of the COVID-19 lockdown, there were not enough UDC hubs open, resulting in some patients travelling long distances, often on public transport. In the event of further spikes or subsequent pandemics, it is important that there is sufficient local provision of services to reduce the risk of virus transmission and improve access for care

- Certain conditions were and can easily be managed over the telephone, negating the need for the patient to travel for a face-toface consultation

- All data collection was on paper which was inefficient and so moving to electronic collection would improve efficiency and allow some clinicians to work from home

- Using 'Attend Anywhere' virtual video conferencing software could allow video consultations to reduce patient footfall, while enabling clinicians to tackle the backlog of patients that arose due to cancellation of elective services. This does require availability of sufficient rooms to maintain confidentiality

- An effort should be made to collect data on whether patients are registered with a dentist, their normal dental attendance record and their deprivation status, as this may be important for dental public health to try and address the oral health inequities in the UK.

\section{Conclusions}

The dental profession has had to make some significant changes as a result of the risk of COVID-19 transmission and due to the effects of the lockdown. With enough UDC hubs, patients will have their emergency conditions managed local to their home address, helping to reduce the spread of the virus. Telephone triage has become an integral part of dentistry throughout the pandemic, ensuring emergency resources are prioritised for those in greatest need. This review has highlighted the effectiveness of triage, as well as its drawbacks. The staff at the secondary care UDC hub worked well together to deliver an effective service for patients during a time of great uncertainty.

\section{Ethics declaration}

The authors declare no conflicts of interest.

\section{References}

1. NHS England and NHS Improvement. Issue 2: Preparedness letter for primary dental care. 2020. Available online at https://www.england.nhs.uk/ coronavirus/publication/preparedness-letters-fordental-care/ (accessed November 2020).

2. Domman M-A. Indicies of Deprivation 2019. 2019. Available at https://www.londoncouncils.gov.uk/ members-area/member-briefings/local-governmentfinance/indices-deprivation-2019 (accessed November 2020) 